\title{
The Optoelectronic Properties of Sb Doped BBi Compounds
}

\author{
M. UstundaG*, B.G. Yalcin, M. Aslan, S. BagCi \\ Sakarya University, Physics Department, Sakarya, Turkey
}

\begin{abstract}
We have determined structural, electronic and optical properties of $\mathrm{BBi}_{1-x} \mathrm{Sb}_{x}$ alloy by using density functional theory based on the full potential linearized augmented plane wave method. For the exchange correlation potential, the generalized gradient approximation of Perdew, Burke, and Ernzerhof has been performed. The structural properties, including lattice constants and bulk modulus have been calculated by changing $x$ concentration. We have investigated the effect of composition on lattice constant, bulk modulus and band gap. Properly, direct/indirect band character of $\mathrm{BBi}_{1-x} \mathrm{Sb}_{x}$ has been investigated depending on the $x$ concentration. Then we have determined some basic linear optical properties $\mathrm{BBi}_{1-x} \mathrm{Sb}_{x}$ alloy in direct band gap region. The obtained results have been compared with available studies. All the calculations have been performed after geometry optimization. As far as we know, no experimental or theoretical data are presently available for the studied ternary alloy $\mathrm{BBi}_{1-x} \mathrm{Sb}_{x}$ $(0<x<1)$.

DOI: 10.12693/APhysPolA.130.98

PACS/topics: 71.15.Mb, 78.20.Ci, 71.20.-b, 61.72.Vv
\end{abstract}

\section{Introduction}

Boron- $\mathrm{V}$ semiconductor alloys have recently received substantial attention due to their applications in electronic and optoelectronic device technology [1-3]. Boron compounds are advisable for variety applications due to their extreme properties such as low ionicity, high thermal conductivity and hardness [4]. BBi and BSb which are the last two members of boron- $\mathrm{V}$ family have attracted increasing research attention theoretically in the recent time. Most of the works were about the structural and electronic properties of $\mathrm{BSb}$ and $\mathrm{BBi}$ compounds [5-11]. In these works researchers have found direct and indirect band gap behaviors for $\mathrm{BBi}$ and $\mathrm{BSb}$, respectively.

Alloying a semiconductor can be suitable to meet specific requirements of researches such as lattice parameter, band gap, and hardness. According to the best of our knowledge neither experimental nor theoretical investigation related to $\mathrm{BBi}_{1-x} \mathrm{Sb}_{x}$ alloy have existed in the literatures. Consequently, for the first time in this investigation, we replaced $\mathrm{Bi}$ with $\mathrm{Sb}$ in $\mathrm{BBi}$ to obtain $\mathrm{BBi}_{1-x} \mathrm{Sb}_{x}$ alloy. In numerous technological applications, it is significant to determine direct to indirect band gap transition concentration for a semiconductor alloy. In this investigation, we have studied structural and electronic properties of $\mathrm{BBi}_{1-x} \mathrm{Sb}_{x}$ alloy at different $x$ concentrations. We have added $6.25 \% \mathrm{Sb}$ as an alternative of $\mathrm{Bi}$ atoms in BBi compounds in every step. Consequently, the effect of little $\mathrm{Sb}$ addition (6.25\%) to BBi is represented. The variance in lattice parameter and bulk modulus is studied. The changes in band gap energy and direct to indirect band gap transition concentration and some optical properties have been also determined. The perturbation owing to $\mathrm{Sb}$ concentration on the valence and conduction bands has been also investigated in this study.

\footnotetext{
*corresponding author; e-mail: mustundag@sakarya.edu.tr
}

\section{Method and calculation}

In the present work, first principles calculations for $\mathrm{BBi}_{1-x} \mathrm{Sb}_{x}$ alloys are performed using the full potential linearized augmented plane wave (FP-LAPW) [12] method based on density functional theory (DFT) as applied in WIEN2k [13] computer package program. The exchange and correlation interactions are taken into account by means of generalized gradient approach (GGA) based on Perdew et al. [14]. In order to get the total energy convergence, we have expanded the Kohn-Sham wave functions up to $R_{\mathrm{MT}} K_{\max }=7$, where $R_{\mathrm{MT}}$ represents the plane wave radii and $K_{\max }$ gives the magnitude of the maximum reciprocal lattice vector. The wave function inside atomic spheres is expanded up to $l_{\max }=10$, while the charge density is Fourier expanded up to $G_{\max }=12 \mathrm{Ry}^{1 / 2}$. To separate core from valence states the cut-off energy is set to $-6 \mathrm{Ry}$. The standard special $k$-points technique of Monkhorst and Pack (MP) [15] is used for accurate Brillouin zone (BZ) integrations. We have used $2 \times 2 \times 5 \mathrm{MP}$ meshes and 9 special $k$-points are taken in the irreducible wedge of the BZ.

\section{Results and discussions}

\subsection{Structural and electronic properties}

First of all, we have analyzed the structural properties of the binary $\mathrm{BBi}$ and $\mathrm{BSb}$ compounds and ternary $\mathrm{BBi}_{1-x} \mathrm{Sb}_{x}$ alloys in zinc blende (ZB) structure utilizing PBE-GGA scheme. Then we have patterned the alloys by 0.0625 increasing $x$ compositions from 0 to 1 . These compositions with ordered structures are selected with periodically repeated $2 \times 2 \times 1$ super-cells with 32 atoms per unit cell. In order to get optimum lattice constants, the total energies calculated as a function of unit cell volume are fitted with the Birch-Murnaghan equation of state [16]. The bulk modulus and band gap of $\mathrm{BBi}_{1-x} \mathrm{Sb}_{x}$ have been calculated by the corresponding equilibrium lattice constants. The calculated lattice parameter (a), bulk modulus $(\mathrm{B})$ and band gap energy $\left(E_{\mathrm{g}}\right)$ of binaries $(\mathrm{BBi}-\mathrm{BSb})$ and ternaries $\left(\mathrm{BBi}_{1-x} \mathrm{Sb}_{x}\right)$ are summarized 
in Table I. The calculated lattice parameter (a) and bulk modulus (B) for BBi and BSb are in good agreement with other theoretical studies [5, 8-12]. The band gap energies and band character (direct/indirect) of the studied materials are represented in the same table. For $\mathrm{BBi}_{1-x} \mathrm{Sb}_{x}$, as a result of our calculations, it has shown direct band gap in the $x$ concentration range between $0 \%$ and $31.25 \%$, while it has shown indirect band gap with the $x$ concentration range between $37.5 \%$ and $100 \%$. It is clearly seen from this table that transition from direct to indirect band gap occurs with $x$ concentration between $31.25 \%$ and $37.5 \%$

TABLE I

The equilibrium lattice constants (a), bulk modulus (B) and band gap energy $\left(E_{\mathrm{g}}\right)$ of $\mathrm{BBi}_{1-x} \mathrm{Sb}_{x}$.

\begin{tabular}{c|c|c|c|c}
\hline \hline $\begin{array}{c}x \% \\
\text { concentration }\end{array}$ & $\begin{array}{c}a \\
{[\AA]}\end{array}$ & $\begin{array}{c}B \\
{[\mathrm{GPa}]}\end{array}$ & $\begin{array}{c}E_{\mathrm{g}} \\
{[\mathrm{eV}]}\end{array}$ & $\begin{array}{c}\text { Direct (D) } \\
\text { Indirect (I) }\end{array}$ \\
\hline & 5.53 & 68.52 & & \\
0 & $5.53[8]$ & $72.2[8]$ & 0.09 & $\mathrm{D}$ \\
& $5.46[9]$ & $81.89[9]$ & $0.14[8]$ & $\mathrm{D}$ \\
\hline 6.25 & $5.44[10]$ & $85.87[10]$ & & \\
12.5 & 5.51 & 71.73 & 0.23 & $\mathrm{D}$ \\
18.75 & 5.48 & 75.70 & 0.36 & $\mathrm{D}$ \\
25 & 5.46 & 78.90 & 0.60 & $\mathrm{D}$ \\
31.25 & 5.45 & 80.60 & 0.73 & $\mathrm{D}$ \\
37.5 & 5.43 & 82.15 & 0.75 & $\mathrm{I}$ \\
43.75 & 5.41 & 83.33 & 0.74 & $\mathrm{I}$ \\
50 & 5.40 & 85.66 & 0.76 & $\mathrm{I}$ \\
56.25 & 5.39 & 87.03 & 0.74 & $\mathrm{I}$ \\
62.5 & 5.37 & 88.10 & 0.76 & $\mathrm{I}$ \\
68.75 & 5.36 & 89.53 & 0.76 & $\mathrm{I}$ \\
75 & 5.34 & 92.05 & 0.76 & $\mathrm{I}$ \\
81.25 & 5.32 & 93.14 & 0.76 & $\mathrm{I}$ \\
87.5 & 5.30 & 94.90 & 0.76 & $\mathrm{I}$ \\
93.75 & 5.29 & 96.09 & 0.76 & $\mathrm{I}$ \\
\hline & 5.27 & 96.43 & 0.76 & $\mathrm{I}$ \\
100 & $5.277[5]$ & $99.5[5]$ & $0.71[10]$ & $\mathrm{I}$ \\
& $5.21[11]$ & $110[11]$ & $0.75[12]$ & $\mathrm{I}$ \\
\hline & & & &
\end{tabular}

In order to obtain $x$ concentration for direct to indirect transition, we have applied linear fit to the $\Gamma-\Gamma$ and $\Gamma-X$ transition energies. As a result, we have found the exact $x$ concentration value at $x=32.03$ for this transition as shown in Fig. 1. The relation between Sb concentrations and band gap energy values can also be shown in the same figure. For the studied materials, the valence band maximum is at $\Gamma$ point while conduction band minimum occurs at $\Gamma$ or $X$ points for direct or indirect transitions, respectively. Replacing $\mathrm{Sb}$ with $\mathrm{Bi}$ increases the $\Gamma-\Gamma$ transition energy linearly. It means that in direct band gap region the band gaps of $\mathrm{BBi}_{1-x} \mathrm{Sb}_{x}$ alloys increase with increasing Sb concentration. It is clearly seen from Fig. 1 that there is no band gap bowing in the direct band gap region. This means that there is no nonlinearity between band gap and $x$ concentration. Therefore, it is consistent with the virtual crystal approximation [17]. On the other hand, in the indirect band gap region, increasing $\mathrm{Sb}$ concentration does not have such effect on the $\Gamma-X$ transition energy.

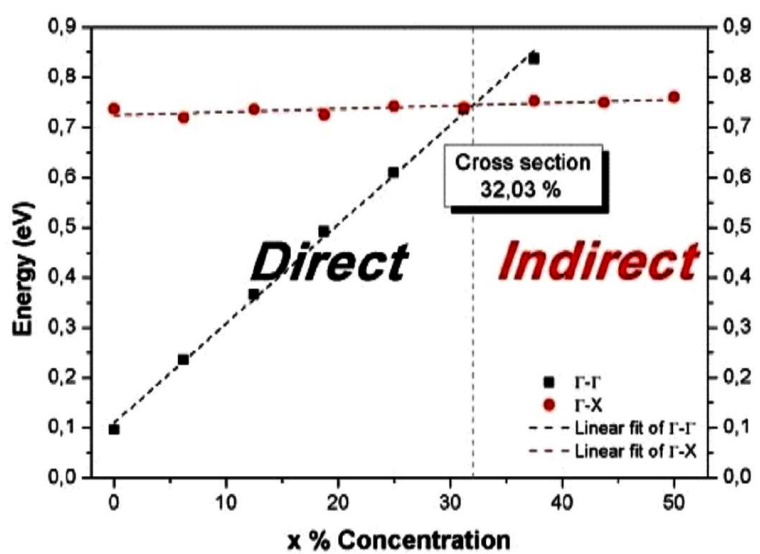

Fig. 1. $\quad \Gamma-\Gamma$ and $\Gamma-X$ transition energies with different $x$ concentrations for studied material.

Figure 2 indicates that increasing Sb concentration has a great effect on the conduction band in the direct band gap region. For $\mathrm{BBi}_{1-x} \mathrm{Sb}_{x}$, the minimum of conduction band energy at $\Gamma$ point increases while Sb concentration is increasing. On the other hand, the change in Sb concentration does not have such effect on the valence band maximum energy. Therefore, this situation affects the mobility of electrons in the conduction bands. Increase of the Sb concentration decreases the material quality due to perturbation on conduction band minimum.

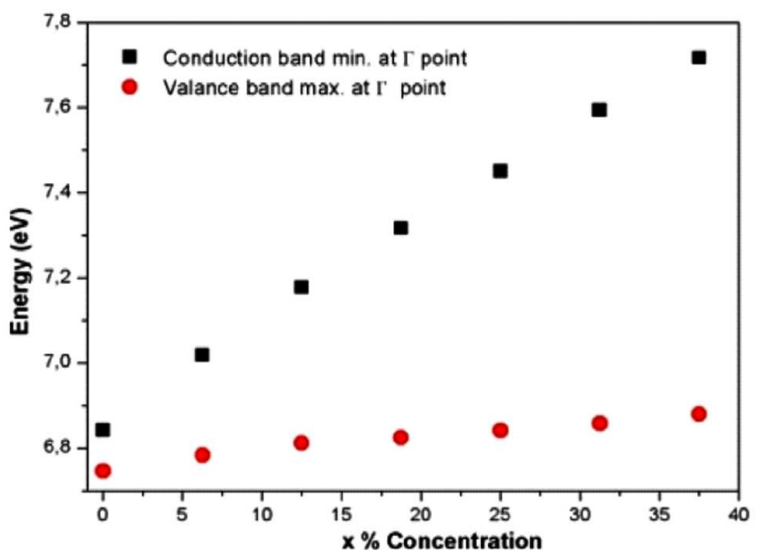

Fig. 2. Valence and conduction band transition energies at $\Gamma$ point with different $x$ concentrations for studied material.

\subsection{Optical properties}

Our calculated imaginary $\varepsilon_{2}(\omega)$ and real $\varepsilon_{1}(\omega)$ parts of the dielectric function for $\mathrm{BBi}_{1-x} \mathrm{Sb}_{x}$ alloys are given in Fig. 3a and b, respectively. Notice first that these compounds have fundamental absorption limit at infrared spectrum region which is important for communication 
devices in technological applications. It is clearly seen from Fig. 3a that $\varepsilon_{2}(\omega)$ curves for $\mathrm{BBi}_{1-x} \mathrm{Sb}_{x}$ alloys (at $x=0.0625,0.125,0.1875,0.25$ ) have shown similar behavior with each other.
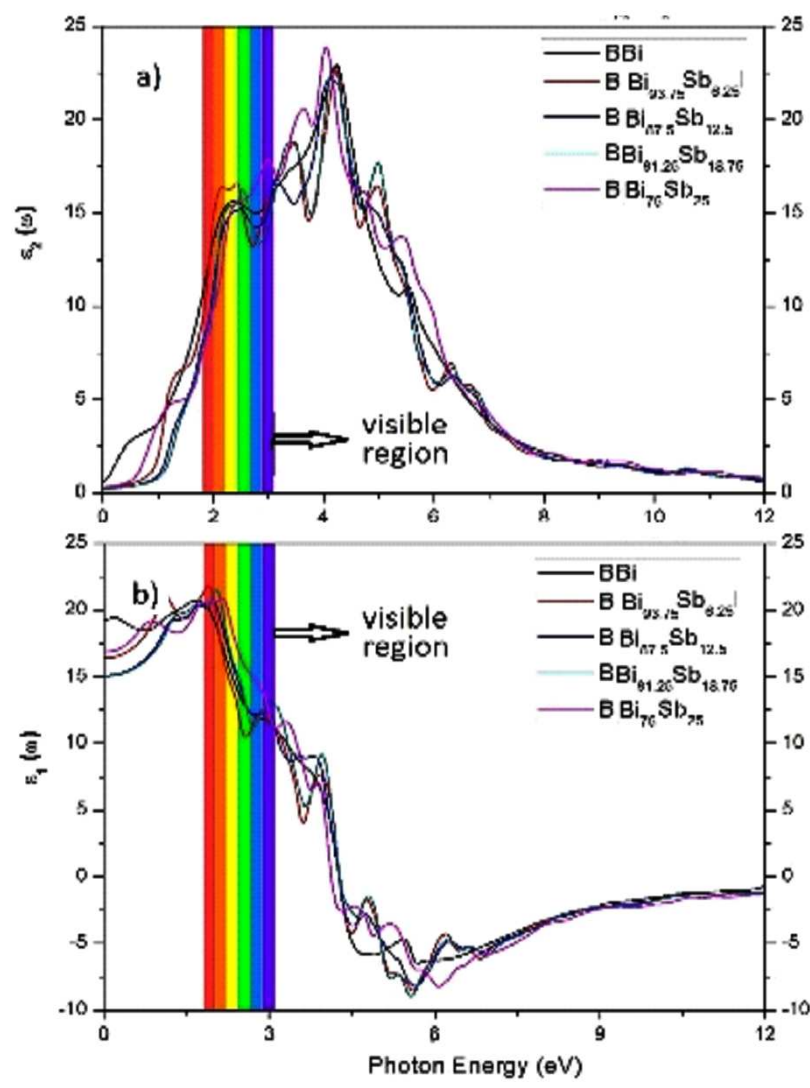

Fig. 3. Calculated (a) imaginary $\varepsilon_{2}(\omega)$ and (b) real $\varepsilon_{1}(\omega)$ parts of the dielectric function.

In this figure, there is one main peak, around 4-4.5 eV for all concentrations. This and other smaller peaks are due to the critical-point transitions. For the detailed analysis of the structures in $\varepsilon_{2}(\omega)$ can be identified with transitions between bands at critical points or along symmetry lines in the BZ. Additionally, because of decreasing trend of $\varepsilon_{2}(\omega)$ the crystals become transparent in highenergy region. From Fig. 3b, the first peaks of $\varepsilon_{1}(\omega)$ stem from the electronic transition from valence to conduction bands and it is also observed that $\varepsilon_{1}(\omega)$ decreases for all concentrations in the visible region.

\section{Conclusion}

In this study, structural and electronic properties of $\mathrm{BBi}_{1-x} \mathrm{Sb}_{x}$ alloys have been determined by using DFT depending on FP-LAPW as applied in the WIEN2k. This work made clear that in direct band gap region, band gaps are consistent with virtual crystal approximation. For $\mathrm{BBi}_{1-x} \mathrm{Sb}_{x}$ alloys, we have found direct to indirect band gap transition concentration with $x=32.03 \%$. Also, it is found that increasing $\mathrm{Sb}$ concentration in
$\mathrm{BBi}_{1-x} \mathrm{Sb}_{x}$ decreases material quality in the direct band region, due to its great effect on the conduction band minimum in this region. Using our structural and electronic calculations, the optical properties of $\mathrm{BBi}_{1-x} \mathrm{Sb}_{x}$ alloy for the direct band gap region have been also given. It is clearly seen from the optical results that in the highenergy region, the crystals become transparent due to decreasing trend of $\varepsilon_{2}(\omega)$ and these materials may be suitable for screening ultraviolet radiations. Therefore, the authors hope that the obtained results can be used to cover the lack of data in the literature for $\mathrm{BBi}_{1-x} \mathrm{Sb}_{x}$ alloy.

\section{Acknowledgments}

This work was supported by the Scientific and Technical Research Council of Turkey (TUBITAK) (Project No. 114F479).

\section{References}

[1] R.M. Wentzcovitch, M.L. Cohen, P.K. Lam, Phys. Rev. B 36, 6058 (1987).

[2] R.M. Wentzcovitch, K.J. Chang, M.L. Cohen, Phys. Rev. B 34, 1071 (1986).

[3] B. Bouhafs, H. Aourag, M. Ferhat, M. Certier, J. Phys. Condens. Matter 11, 5781 (1999).

[4] B.G. Yalcin, Physica B 462, 64 (2015).

[5] M. Ustundag, M. Aslan, B.G. Yalcin, Comput. Mater. Sci. 81, 471 (2014).

[6] B.G. Yalcin, S. Bagci, M. Ustundag, M. Aslan, Comput. Mater. Sci. 98, 136 (2015).

[7] A. Zaoui, F. El Haj Hassan, J. Phys. Condens. Matter 13, 253 (2001).

[8] D. Madouri, M. Ferhat, Phys. Status Solidi B 242, 2856 (2005)

[9] S. Cui, W. Feng, H. Hu, Z. Feng, Y. Wang, Comput. Mater. Sci. 47, 968 (2010).

[10] K. Amara, B. Soudini, D. Rached, A. Boudali, Comput. Mater. Sci. 44, 635 (2008).

[11] D. Varshney, G. Joshi, M. Varshney, S. Shriya, Physica B 405, 1663 (2010).

[12] J.C. Slater, Adv. Quant. Chem. 1, 35 (1964).

[13] P. Blaha, K. Schwarz, G. Madsen, D. Kvasnicka, J. Luitz, An Augmented Plane Wave plus Local Orbital Program for Calculating the Crystal Properties, 12th ed., WIEN, 2012.

[14] J.P. Perdew, K. Burke, M. Ernzerhof, Phys. Rev. Lett. 7, 3865 (1996).

[15] H.J. Monkhorst, J.D. Pack, Phys. Rev. B 13, 5188 (1976).

[16] F. Birch, Phys. Rev. 71, 809 (1947).

[17] A. Sommerfeld, Naturwissenschaften 22, 49 (1934). 